\title{
Multiple neonicotinoids in children's cerebro-spinal fluid, plasma, and urine
}

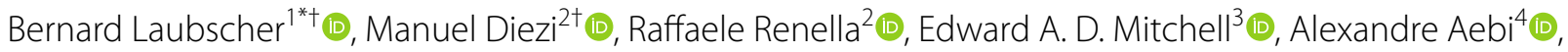 \\ Matthieu Mulot ${ }^{3}$ [D and Gaëtan Glauser ${ }^{5}$ (D)
}

\begin{abstract}
Background: Neonicotinoids (NN) are selective neurotoxic pesticides that bind to insect but also mammal nicotinic acetycholine receptors (nAChRs). As the most widely used class of insecticides worldwide, they are ubiquitously found in the environment, wildlife, and foods, and thus of special concern for their impacts on the environment and human health. nAChRs are vital to proper brain organization during the prenatal period and play important roles in various motor, emotional, and cognitive functions. Little is known on children's contamination by NN. In a pilot study we tested the hypothesis that children's cerebro-spinal fluid (CSF) can be contaminated by NN.
\end{abstract}

Methods: NN were analysed in leftover CSF, blood, and urine samples from children treated for leukaemias and lymphomas and undergoing therapeutic lumbar punctions. We monitored all neonicotinoids approved on the global market and some of their most common metabolites by ultra-high performance liquid chromatography-tandem mass spectrometry.

Results: From August to December 2020, 14 children were consecutively included in the study. Median age was 8 years (range 3-18). All CSF and plasma samples were positive for at least one NN. Nine (64\%) CSF samples and 13 (93\%) plasma samples contained more than one NN. Thirteen (93\%) CSF samples had N-desmethyl-acetamiprid (median concentration 0.0123 , range $0.0024-0.1068 \mathrm{ng} / \mathrm{mL}$ ), the major metabolite of acetamiprid. All but one urine samples were positive for $\geq$ one NN. A statistically significant linear relationship was found between plasma/urine and CSF N-desmethyl-acetamiprid concentrations.

Conclusions: We have developed a reliable analytical method that revealed multiple NN and/or their metabolites in children's CSF, plasma, and urine. Our data suggest that contamination by multiple NN is not only an environmental hazard for non-target insects such as bees but also potentially for children.

Keywords: Neonicotinoid, N-desmethyl-acetamiprid, Pesticide, Child, Cerebro-spinal fluid

\section{Background}

In 2017, United Nations rapporteurs called for a new global treaty to regulate and phase out the use of hazardous pesticides in farming as chronic exposure to

\footnotetext{
*Correspondence: Bernard.laubscher@chuv.ch

${ }^{\dagger}$ Bernard Laubscher and Manuel Diezi are shared authorship.

${ }^{1}$ Departments of Paediatrics, Réseau Hospitalier Neuchâtelois, Neuchâtel,

and Lausanne University Hospital and Lausanne University, Lausanne, Switzerland

Full list of author information is available at the end of the article
}

pesticides had been linked to various neurological disorders. Pregnant women, foetuses, and children were also considered as particularly vulnerable [1].

Residential, household or parental exposures to pesticides have been associated with young adult brain tumors, respectively childhood leukemia and paediatric non-central nervous system solid tumors [2-5].

Among pesticides, neonicotinoids (NN), which are selectively neurotoxic and bind to nicotinic acetycholine receptors (nAChRs), are of special concern for their impacts on the environment and human health since they original author(s) and the source, provide a link to the Creative Commons licence, and indicate if changes were made. The images or other third party material in this article are included in the article's Creative Commons licence, unless indicated otherwise in a credit line to the material. If material is not included in the article's Creative Commons licence and your intended use is not permitted by statutory regulation or exceeds the permitted use, you will need to obtain permission directly from the copyright holder. To view a copy of this licence, visit http://creativecommons.org/licenses/by/4.0/. The Creative Commons Public Domain Dedication waiver (http://creativeco mmons.org/publicdomain/zero/1.0/) applies to the data made available in this article, unless otherwise stated in a credit line to the data. 
are the most widely used class of insecticides worldwide [6] and are ubiquitously found in the environment [7], wildlife [8], and various foods $[9,10]$. NN use has been restricted in some part of the world due to their significant toxicity to non-target insects such as bees [11].

In mammals, nAChRs are vital to proper brain organization during the prenatal period [12]. In addition, they play important roles in various motor, emotional, and cognitive functions [12-14]. Little is known on the effect of chronic human low-level exposure to nAChRs' disrupters such as NN, which, especially in human foetuses' and children's developing brains, could potentially lead to later cerebral dysfunctions. In humans, NN have been associated with small-for-gestational-age neonates, congenital malformations, autism spectrum disorder, memory loss and finger tremor [15-19]. NN toxicological studies in rodents or mammals/human cell-lines have been shown to be cytotoxic, genotoxic, hepatotoxic, haematotoxic, nephrotoxic and potentially immunotoxic [20-23]. Among pesticides, NN definitely represent a potential significant public-health risk.

Data on NN distribution and metabolism in humans are scarce [24]. There are very few studies in children: six publications on NN urinary levels [19, 25-29] and one study on NN hair concentrations (6-83 years old people, of whom 28 were $<16$ years) [30]. In China, NN residues in urine were found in $81 \%$ of 289 seven to 11 year old school children [29] and in $100 \%$ of 324 tested donors of all ages (1-97 years, 111 children < 18 years) [25]. In Japan, urinary $\mathrm{N}$-desmethyl-acetamiprid (Desm-ACT) was detected in $14 / 57(24.6 \%)$ very low birth weight infants within $48 \mathrm{~h}$ of birth [19]. This level of prevalence is worrying and calls for studies on other body compartments and on potential health impacts. There are no data on direct human brain exposure to $\mathrm{NN}$ and there is only one report on NN detection in human cerebrospinal fluid (CSF), in a post mortem of a male adult after voluntary and fatal imidacloprid ingestion [31]. Assessing NN presence in children's brain tissue is ethically complicated and, currently, the best available surrogate for brain environment is CSF [32].

The general aims of this pilot study were to develop an analytical protocol to measure NN in children's CSF and to assess if NN are present in children's CSF. Our hypothesis was that NN could be found in children's CSF, thereby representing a potential central nervous system (CNS) exposure.

\section{Methods}

In a prospective paediatric observational study in Switzerland, we conducted a comparative analysis of CSF, blood and urine to develop and test the analytical method and compare the measured concentrations in these three body fluids.

\section{Patients and procedures}

To study children's CSF in an ethically acceptable way, we included, as a convenience sample, children with oncologic disease whose CSF had to be removed for clinical reasons in large enough volumes to allow aliquots to be collected for research purposes. All children were diagnosed and treated at the Department of paediatrics, Lausanne University Hospital, where they were consecutively included between August and December 2020. After initial diagnostic procedures, all children (0-18years) presenting acute lymphoblastic (ALL), myeloblastic (AML) leukaemia or non-Hodgkin lymphoma (NHL) and were planned to receive routine intrathecal chemotherapy were approached and included in the study after informed consent was obtained. Intrathecal treatments consisted in removing up to $8 \mathrm{~mL}$ of CSF by lumbar tap under conscious sedation or anaesthesia before injecting $8-15 \mathrm{~mL}$ of various chemotherapeutic agents; two $\mathrm{mL}$ of CSF was reserved for routine chemical, biological, and cytological investigations and $2 \mathrm{~mL}$ of the leftover CSF was collected for NN analysis. On the same occasion, blood was drawn for clinical purposes from a subcutaneous implantable device. From the four $\mathrm{mL}$ drawn to rinse the device (internal volume $\leq 1.7 \mathrm{~mL}$ ), the first $2 \mathrm{~mL}$ were discarded, and the following $2 \mathrm{~mL}$ were kept (3.2\% Citrate S-Monovette ${ }^{\circledR}$, Sarstedt AG, Sevelen Switzerland) for NN analysis. The patient also voided freely a $5-10 \mathrm{~mL}$ aliquot of urine in a clean tube. CSF and urine were initially kept in $15 \mathrm{~mL}$ polypropylene tubes. All samples were centrifuged, to collect plasma from the blood samples and to remove sediments from the CSF and urine samples. The supernatants were stored at $-80^{\circ} \mathrm{C}$.

\section{NN analysis}

Aliquots of $0.2 \mathrm{~mL}$ of CSF, plasma or urine were placed into $2.0 \mathrm{~mL}$ microcentrifuge tubes, to which $580 \mu \mathrm{L}$ of acetonitrile and $20 \mu \mathrm{L}$ of a solution of isotopically labelled internal standards (IS) were added. The IS solution contained acetamiprid-d3 (CDN Isotopes, Pointe-Claire, Canada), clothianidin-d3 (CDN Isotopes), thiamethoxam-d4 (CDN Isotopes), thiacloprid-d4 (CDN Isotopes), imidacloprid-d4 (CDN Isotopes), dinotefuran-d3 (EQ Laboratories, Augsburg, Germany), and nitempyram-13C-d3 (Alsachim, Illkirch-Graffenstaden, France) at a concentration of $50 \mathrm{ng} / \mathrm{mL}$ in acetonitrile. The mixture was swiftly vortexed and ultrasonicated to precipitate proteins. After centrifugation, the supernatant was partially evaporated to a volume of ca. $50-100 \mu \mathrm{L}$, diluted with $950 \mu \mathrm{L}$ of a $2 \%$ formic acid solution in water and submitted to a purification procedure using solid-phase 
extraction (SPE) cartridges. The SPE cartridge was first conditioned with $1 \mathrm{~mL}$ of methanol, equilibrated with $1 \mathrm{~mL}$ of $2 \%$ formic acid, loaded with the sample, washed with $1 \mathrm{~mL}$ of $0.1 \%$ formic acid and finally eluted with $1 \mathrm{~mL}$ of methanol. The fraction was evaporated to dryness and reconstituted in $200 \mu \mathrm{L}$ of methanol $25 \%$ in water, ultrasonicated, centrifuged, and filtered through $13 \mathrm{~mm}$ hydrophilic PTFE filters. A $2.5 \mu \mathrm{L}$ of the resulting extract was injected into an ultra-high performance liquid chromatography-tandem mass spectrometry (UHPLC-MS/MS) system composed of an Acquity UPLC I-Class and a TQ-XS triple quadrupole (Waters, Milford, MA). NN were detected and quantified according to Kammoun and colleagues ${ }^{34}$. All neonicotinoids and related insecticides approved on the global market and their most commonly detected metabolites were targeted: acetamiprid, clothianidin, thiamethoxam, thiacloprid, imidacloprid, dinotefuran, nitempyram, sulfoxaflor, flupyradifurone, Desm-ACT, imidacloprid-olefin (IMIolefin), desnitro-imidacloprid (IMI-NH), and 6-chloronicotinic acid (6-CAN). The column used for the separation was an Acquity UPLC HSS T3 $(2.1 \times 100 \mathrm{~mm}$, Waters). Mobiles phases were milli-Q water $+0.05 \%$ formic acid $+1 \mathrm{mM}$ ammonium formate (phase A) and acetonitrile $+0.05 \%$ formic acid (phase B). The gradient program started at $0 \%$ phase B and increased linearly to $37.5 \%$ in $7.5 \mathrm{~min}$, followed by a rapid increase to $100 \% \mathrm{~B}$ in $0.5 \mathrm{~min}$, wash at $100 \%$ B for 2 min and reequilibration at $0 \%$ B for $4 \mathrm{~min}$. The MS source conditions were identical to those presented in Kammoun and colleagues [33] except that the MS was operated in both electrospray positive and negative ionization using a $20 \mathrm{~ms}$ polarity switching time. Neonicotinoids were monitored based on the multiple reaction monitoring (MRM) mode. Compound-dependent parameters are presented in supplementary Table 1.

\section{Method validation}

The performances of the method in terms of precision (expressed as \% relative standard deviation (RSD)) and accuracy (expressed in \%) were evaluated by spiking a known amount of $\mathrm{NN}$ in the different matrices before sample preparation (final concentration of $1 \mathrm{ng} / \mathrm{mL}$, $n=4$ for each matrix). We could not perform validation at multiple concentrations due to too restricted sample availability. Coefficients of variations (\%RSD) were below $10 \%$ for all analytes and accuracies always ranged between 80 and $110 \%$ except for desnitro-imidacloprid in CSF and imidacloprid-olefin in plasma for which lower but still acceptable accuracy values of 74.1 and $67.1 \%$, respectively, were obtained (supplementary Table 2). The response function was established using 6-point calibration curves ranging between 0.005 and $10 \mathrm{ng} / \mathrm{mL}$. Linear or quadratic curves weighted by $1 / \mathrm{x}$ were applied. Limits of quantification (LOQs) were evaluated as the concentrations giving signal-to-noise ratios of 10 in spiked samples. The method LOQs in CSF ranged between 1 and $20 \mathrm{pg} / \mathrm{mL}$ for all substances except 6-CAN for which the LOQ was $200 \mathrm{pg} / \mathrm{mL}$. Plasma LOQs were similar to CSF's but urine LOQs were generally higher due to increased background noise and matrix effects (supplementary Table 2). The specificity was determined by measuring blank solutions submitted to the entire preparation as well as spiked samples.

\section{Total CSF protein content}

The total CSF protein concentration was measured using a pyrogallol red-based colorimetric assay (Randox Laboratories, Crumlin, UK) on a Cobas $8000^{\circledR}$ modular analyzer (Roche Diagnostics, Rotkreuz, Switzerland).

\section{Specific gravity}

Urinary concentrations can vary widely over the period of elimination of xenobiotics and largely depend on fluid intake. The two most common methods for correction of metabolite concentrations in urine are the measure of specific gravity and that of creatinine [34]. The specific gravity of urine samples was measured using a digital Atago PEN urine specific gravity refractometer (Tokyo, Japan). In brief, an aliquot of urine $(0.2 \mathrm{~mL})$ was placed on a microscope slide and the pen tip put horizontally on the slide for about 2 seconds until the measurement was done.

\section{Statistical analyses}

Statistical analyses were carried out in R statistical software [35]. To assess the relationship between NN concentration is CSF and plasma or urine, we first selected NNs for which the number of samples with a concentration above LOQ was greater than 10. We then computed the association between NN concentration in CSF vs plasma and CSF vs urine using standard OLS linear regressions. Regressions were built using the $\mathrm{lm}$ function from the stats package [36] through ggpubr [37]. We set alpha $=0.05$ as threshold for any analysis.

As this pilot study was only meant to test the feasibility of the NN measurements in children's CSF, the only patient's characteristics that were obtained were genders and ages and they were not considered as relevant confounding factors from a medical standpoint.

The study was approved by our local Institutional Review Board (Commission cantonale d'éthique de la recherche sur l'être humain, CER-VD). All teenagers/parents gave their written consent. 


\section{Results}

Over a 4 months period, 14 children with ALL $(n=9)$ or AML $(n=2)$ or NHL $(n=3)$ were consecutively included. $\mathrm{M} / \mathrm{F}$ ratio was 1.0 , median age 8 years (range 3-18). All CSF total protein contents were within institutional normal ranges $(150-450 \mathrm{mg} / \mathrm{l})$.

All CSF presented detectable levels of at least one NN (Desm-ACT in 14/14, sulfoxaflor in 7/14, thiamethoxam in 6/14, and imidacloprid 2/14) (see Table 1 or supplementary Table 3 for detailed results). Five CSF contained a single NN (all with Desm-ACT), three contained two (two with Desm-ACT and sulfoxaflor and one with Desm-ACT and thiamethoxam), and six contained three (four with Desm-ACT, sulfoxaflor and thiamethoxam, one with Desm-ACT, sulfoxaflor and thiamethoxam and one with Desm-ACT, sulfoxaflor and imidacloprid). Thirteen out of 14 CSF had quantifiable levels of Desm-ACT (median concentration 0.0123 , range $0.0024-0.1068 \mathrm{ng} / \mathrm{mL}$ ). Four out of 14 CSF had quantifiable levels of sulfoxaflor (median concentration 0.0053 , range $0.0024-0.0124 \mathrm{ng} / \mathrm{mL}$ ). Three out of $14 \mathrm{CSF}$ had quantifiable levels of thiamethoxam (median concentration 0.0196, range $0.0054-0.0765 \mathrm{ng} /$ $\mathrm{mL}$ ). One out of $14 \mathrm{CSF}$ had quantifiable level of imidacloprid $(0.0153 \mathrm{ng} / \mathrm{mL})$. All plasma samples were positive for at least one $\mathrm{NN}$ : one with only thiamethoxam, six with two (three with sulfoxaflor and Desm$\mathrm{ACT}$, two with imidacloprid and Desm-ACT, one with thiamethoxam and Desm-ACT), five with three (two with thiametoxam, sulfoxaflor and Desm-ACT, two with thiametoxam, imidacloprid and Desm-ACT, one with imidacloprid, sulfoxaflor and Desm-ACT) and two with four (both with thiamethoxam, imidacloprid, sulfoxaflor and Desm-ACT). Thirteen out of 14 plasma samples had Desm-ACT (12 of which with quantifiable levels, median concentration 0.0213, range 0.0039$0.1812 \mathrm{ng} / \mathrm{mL}$ ). All but one urine samples were positive for at least one NN (seven with one, four with two, one with three and one with four). Thirteen out of 14 urines had Desm-ACT (median concentration 0.1925, range $0.0378-6.774 \mathrm{ng} / \mathrm{mL})$. Significant linear relationships were found between CSF and plasma/urine DesmACT concentrations (see Fig. 1). Other NN did not meet the inclusion criteria of a minimum of 10 samples with a concentration above LOQ. One data point had a Cook distance greater than one in both models (CSF vs plasma and CSF vs urine), with a Desm-ACT concentration in urine greater than $6 \mathrm{ng} / \mathrm{mL}$. We computed new models excluding this point that were very similar to the full ones, with the exception that the intercept became not significant. All models were significant. Full details on the models are presented in supplementary material (supplementary Figs. 1 and 2, supplementary Tables 4 and 5).

\section{Discussion}

Our study offers two new perspectives on paediatric public-health research: First, it shows that NN detection in children CSF is technically feasible. Second, it reveals that multiple NN can contaminate children's CSF and that, in the case of Desm ACT, plasma and - to a lesser extent urine concentrations correlate with CSF concentrations.

To our knowledge, no study has reported a method for the analysis of low concentrations of NNs in human CSF and their comparison with those in plasma and urine from the same individuals. Thus, we first had to develop a reliable methodology to measure neonicotinoids and their metabolites in these human fluids. The use of very small left-over volumes $(0.2 \mathrm{~mL})$ alleviates the difficulties related to CSF collection. However, starting from small sample volumes may have an impact on positive detection rates, which are often correlated to the method sensitivity [10]. Therefore, a sensitive analytical protocol able to detect traces of $\mathrm{NN}$ had to be developed. Using a combination of a two-step efficient clean-up (protein precipitation followed by solid-phase extraction) and state-of-the-art analysis, we were able to set quantification limits in CSF and plasma ranging between 1 and $20 \mathrm{pg} / \mathrm{mL}$ for all $\mathrm{NN}$ and their metabolites but one (6-CAN). In urine, LOQs generally ranged between 5 and $50 \mathrm{pg} / \mathrm{mL}$, which is comparable or better than those from recent studies that started from higher or similar volumes of urine [19, 27, 38, 39]. In accordance with previous paediatric studies, our work also shows the importance to monitor as many molecules as possible, including the metabolites of parent pesticides [19, 27, 28, 38]. Had we chosen not to measure Desm-ACT, 7/14 children's CSF would have been free of any NN, thus falsely minimizing their degree of contamination by NN. In children, only four studies reported on urinary Desm-ACT median concentrations which ranged between $0.048 \mathrm{ng} / \mathrm{mL}$ and $1.35 \mathrm{ng} / \mathrm{mL}[19,27,28,38]$. Our values (median concentration of $0.19 \mathrm{ng} / \mathrm{mL}$ ) thus corroborate those found in earlier studies. We are not aware of any other studies on NN levels in paediatric body fluids. In 2021, Xu et al. reported on paired urine and blood NN profiles in 196 healthy young adults from China. They found urinary and blood Desm-ACT median concentrations of 0.35 and $0.58 \mathrm{ng} / \mathrm{mL}$ respectively, which are similar for urine but much higher for plasma than those found in our subjects (0.32 and $0.02 \mathrm{ng} / \mathrm{mL}$, respectively) [40].

Our findings suggest that ALL, AML or NHL children living in Switzerland are exposed to multiple NN. Our study population was small and highly selected and is thus not representative of a large paediatric population, 


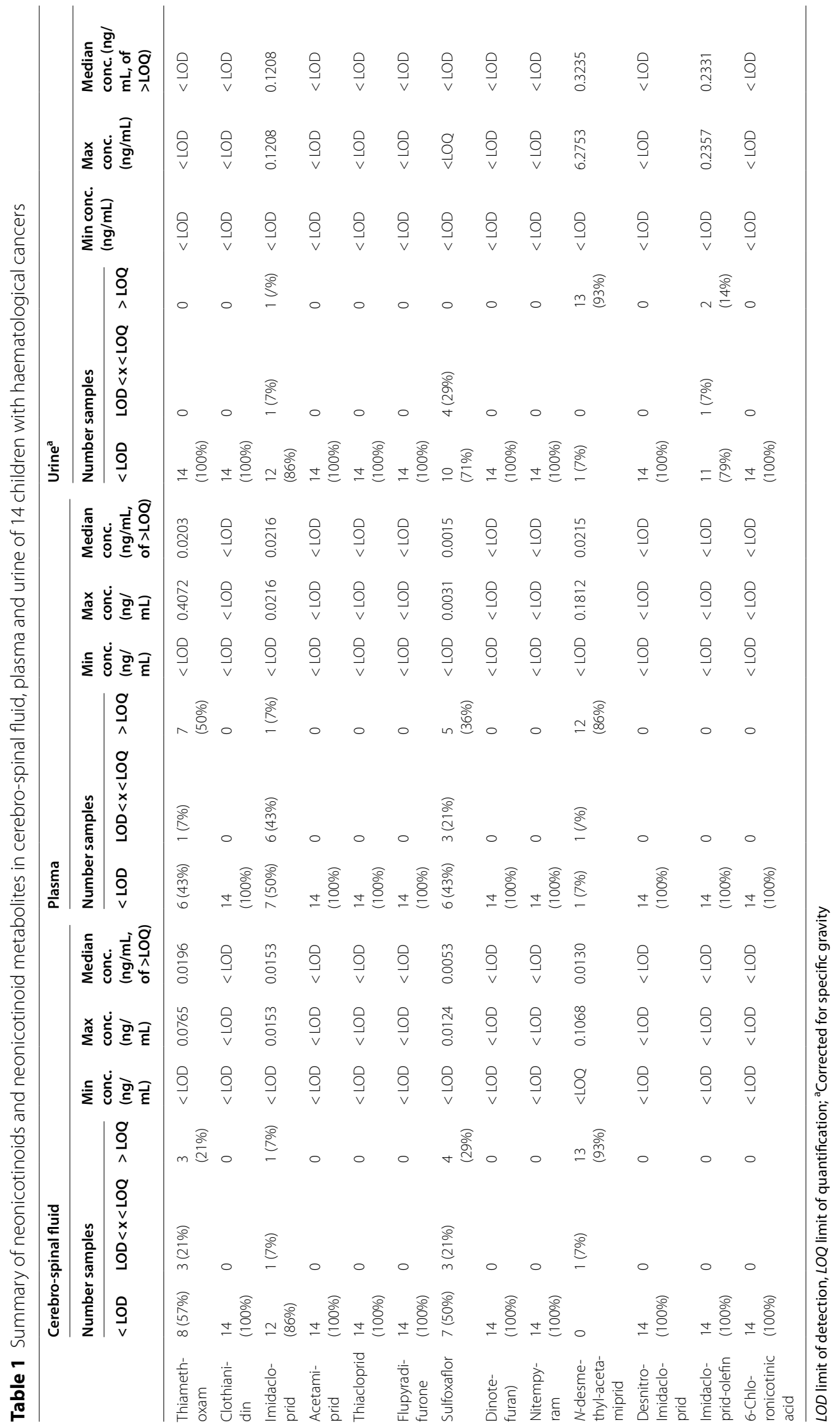



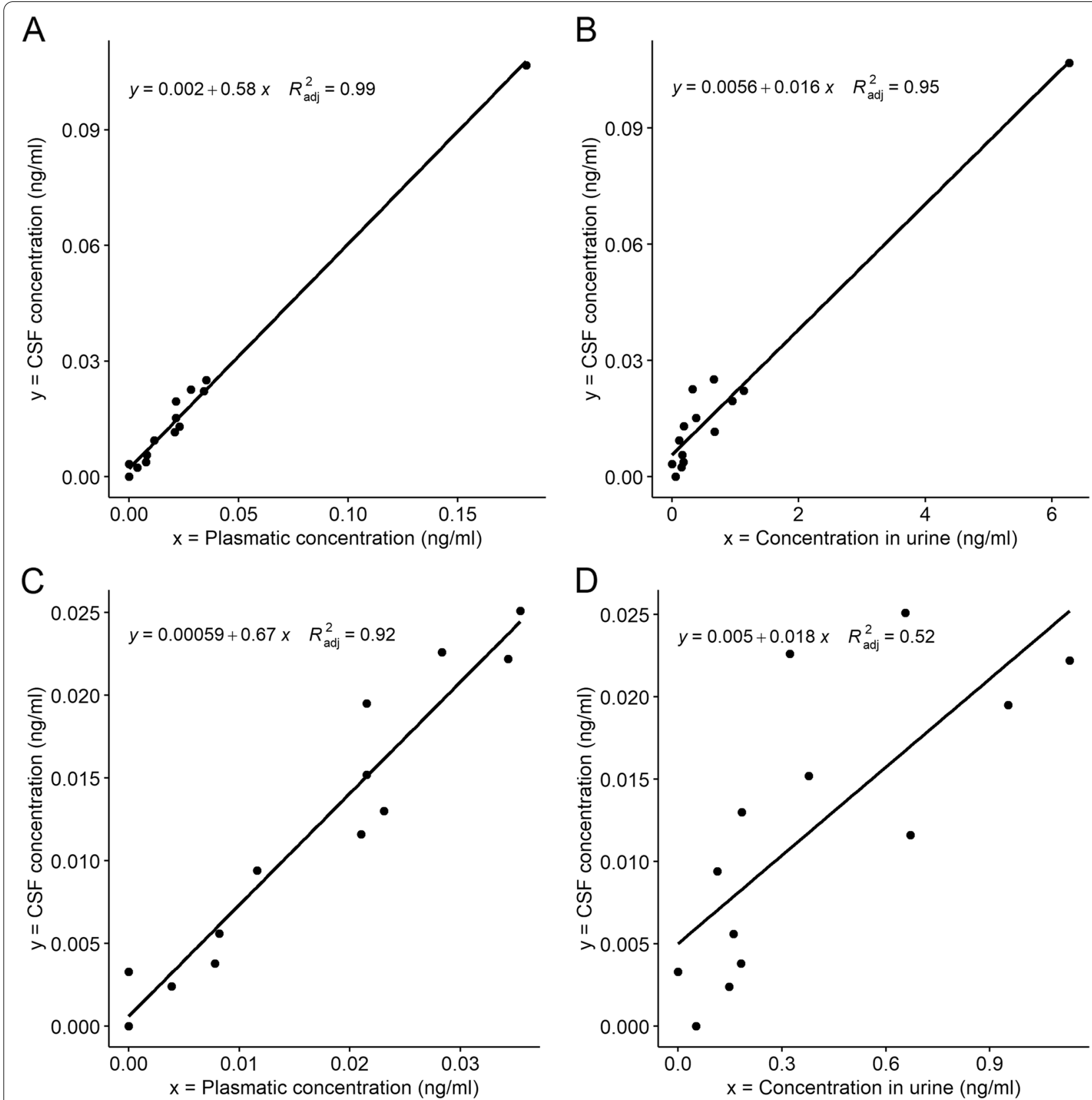

Fig. 1 Cerebro-spinal fluid versus plasma (A and $\mathbf{C}$ ) or urine (B and $\mathbf{D}) \mathrm{N}$-desmethyl-acetamiprid concentrations (ng/ml) of 14 children with haematological cancers. CSF, cerebro-spinal fluid. $\mathbf{A}$ and $\mathbf{B} n=14$. C and $\mathbf{D} n=13$, point with Cook distance $>1$ removed. Urine concentrations corrected for specific gravity

with or without a haematological cancer. As no control population of healthy children was included, we could not analyse if these multiple exposures to $\mathrm{NN}$ were specific to children with leukaemia/lymphoma. As we did not study potential exposures to any dietary pesticides by food or residential proximity to $\mathrm{NN}$ treated crops/livestock/pets, we could not analyse if these multiple exposures to $\mathrm{NN}$ were associated with increased individual environmental risks. The exposure to multiple $\mathrm{NN}$ in all included patients could represent the tip of the iceberg of a larger, but not analysed, exposure to pesticides. Although the Environmental Protection Agency has classified acetamiprid, clothianidin, dinotefuran, imidacloprid, and thiamethoxam as "Not Likely to be Carcinogenic to Humans" [41], and whilst one could argue that chronic exposure in children is too short to raise risks of 
secondary, non-genetic, cancers, exposures to pesticides - as a broad toxicological group - have been associated with children haematological cancer ${ }^{3,4}$ or CNS/non CNS solid tumors ${ }^{2,5}$. Such exposome outcomes to pesticides hazards have also recently been reported for neurodevelopmental issues [42].

To our knowledge, this is the first study on the presence of NN in humans' CSF. Surprisingly, we observed remarkable differences in concentrations between acetamiprid and its metabolite Desm-ACT in CSF. We have found no data on absorption and distribution of $\mathrm{NN}$ and their metabolites in humans. Acetamiprid and Desm$\mathrm{ACT}$ are structurally very similar and have comparable hydrophobicity (calculated $\log P 1.4$ and 1.2, respectively), thus they should theoretically be found in the same body compartments. While we cannot totally exclude a DesmACT specific blood-to-CSF transfer, the observed differences in concentrations may be better explained by a prolonged half-life of Desm-ACT compared to ACT in the context of low-dose chronic exposure, resulting in the detection of the former only. The normal CSF total protein concentrations in the 14 studied patients argues against a meningeal inflammation and, thus, against an increased blood-to-CSF permeability such as in bacterial meningitis [43].

\section{Conclusions}

Our work describes a reliable analytical procedure to measure NN in human CSF. Our data show that children with ALL, AML and NHL living in Switzerland are exposed to multiple NN. They also show that Desm-ACT freely diffuses into children's CSF and that other NN than Desm-ACT are to be found in childrens' CSF. No data exist on human blood-to-brain NN transfer, via the BBB or CSF-to-brain trans-neuroependymal route. The latter's ultrastructure and ontogeny [44] as well as the now established presence of NN in children's CSF warrants caution about a potential direct effect of $\mathrm{NN}$ on the CNS. The vulnerability of the maturing brain to NN could lie in a potential chronic low-dose exposure during a very susceptible developmental period. Exposome medium-term studies, from foetal life to late childhood, similar to the Helix cohort [45], are needed to better delineate potential links between NN exposures and childhood cancers. Organophosphate pesticides were measured in two subgroups of the Helix cohort: it could be meaningful to add NN determination in the same samples. A larger study to collect more CSF/plasma/urine samples in children could yield more data on various $\mathrm{NN}$ levels in CSF/plasma/ urine to allow a sounder analysis of blood-to-CSF or urine-to-CSF NN levels ratio.

\section{Abbreviations}

CNS: Central nervous system; CSF: Cerebro-spinal fluid; Desm-ACT: N-desmethyl-acetamiprid; IMI-NH: Desnitro-imidacloprid; IMI-olefin: Imidacloprid-olefin; NN: Neonicotinoids; 6-CAN: 6-chloronicotinic acid.

\section{Supplementary Information}

The online version contains supplementary material available at https://doi. org/10.1186/s12940-021-00821-z.

Additional file 1: Supplementary Table 1. UHPLC-MS/MS compoundspecific parameters used for the quantification of neonicotinoids. RT, retention time; $\mathrm{ESI}$, electrospray; $\mathrm{CV}$, cone voltage; $\mathrm{CE}$, collision energy.

Additional file 2: Supplementary Table 2. Validation parameters for the different analytes in the different matrices. IS; internal standard; LOQ limit of quantification; CSF cerebrospinal fluid.

Additional file 3: Supplementary Table 3. Detailed results of neonicotinoids and neonicotinoid metabolites in cerebro-spinal fluid, plasma and urine of 14 children with haematological cancers. CSF, cerebro-spinal fluid; LOD, limit of detection; LOQ, limit of quantification; * Corrected for specific gravity.

Additional file 4: Supplementary Figure 1. (CSF occurence). Number of samples with neonicotinoids > lower limit of quantification, per neonicotinoid, in cerebrospinal fluid in 14 children with haematological cancers. NN, neonicotinoid; CSF, cerebro-spinal fluid; LOQ, lower limit of quantification.

Additional file 5: Supplementary Figure 2. (plasma occurence). Number of samples with neonicotinoids > lower limit of quantification, per neonicotinoid, in plasma in 14 children with haematological cancers. NN, neonicotinoid; LOQ, lower limit of quantification.

Additional file 6: Supplementary Table 4. (models summary). Summary statistics of the regression models, with or without extreme value (cook distance $>1$ ).

Additional file 7: Supplementary Table 5. (models coefficients). Coefficient and associated $p$-values of the regression models, with or without extreme value (Cook distance $>1$ ).

\section{Acknowledgements}

We thank all children and their families for their participation in the study.

\section{Authors' contributions}

BL was the principal investigator, designed and planned the study, and wrote the first manuscript draft. MD participated in the design and planning of the study, obtained consent from patients and families, collected and analysed data and participated in manuscript writing. RR helped for the study design, specimen initial collection and management and participated in manuscript writing. EM and AA participated in the study design, data analysis, and participated in manuscript writing. MM performed the statistics and participated in manuscript editing. GG participated in the study design, performed the NN analyses, data analysis, and participated in manuscript writing. The authors read and approved the final manuscript.

\section{Funding}

Lausanne University Hospital, University of Neuchâtel, Stichting Triodos Foundation (The Netherlands), for the Institutional Review Board fees, NN analysis costs, and Open Source publication fees if relevant.

\section{Availability of data and materials}

All data generated or analyzed during this study are included in this published article and its supplementary information files (Additional file, detailed results of NN analysis in CSF, plasma and urine). 


\section{Declarations}

\section{Ethics approval and consent to participate}

The study was approved by our local Institutional Review Board (Commission cantonale d'éthique de la recherche sur l'être humain, CER-VD). All teenagers/ parents gave their written consent.

\section{Consent for publication}

Not applicable.

\section{Competing interests}

AA and EM are members of the IUCN Task Force on Systemic Pesticides.

\section{Author details}

'Departments of Paediatrics, Réseau Hospitalier Neuchâtelois, Neuchâtel, and Lausanne University Hospital and Lausanne University, Lausanne, Switzerland. ${ }^{2}$ Department of Paediatrics, Lausanne University Hospital and Lausanne University, Lausanne, Switzerland. Institute of Biology, University of Neuchâtel, Neuchâtel, Switzerland. ${ }^{4}$ Institutes of Biology and Anthropology, University of Neuchâtel, Neuchâtel, Switzerland. ${ }^{5}$ Neuchâtel Platform of Analytical Chemistry, University of Neuchâtel, Neuchâtel, Switzerland.

Received: 27 July 2021 Accepted: 20 December 2021

Published online: 11 January 2022

\section{References}

1. United Nations. Report of the Special Rapporteur on the right to food. 2017. [Internet]. [cited 2021 Mar 15]. Available from: https://undocs. org/A/HRC/34/48

2. Maele-Fabry GV, Gamet-Payrastre L, Lison D. Residential exposure to pesticides as risk factor for childhood and young adult brain tumors: A systematic review and meta-analysis. Environ Int. 2017;106:69-90.

3. Chen M, Chang C-H, Tao L, Lu C. Residential exposure to pesticide during childhood and childhood cancers: A Meta-analysis. Pediatrics. 2015;136:719-29.

4. Maele-Fabry GV, Gamet-Payrastre L, Lison D. Household exposure to pesticides and risk of leukemia in children and adolescents: updated systematic review and meta-analysis. Int J Hyg Environ Health. 2019;222:49-67.

5. Coste A, Bailey HD, Kartal-Kaess M, Renella R, Berthet A, Spycher BD. Parental occupational exposure to pesticides and risk of childhood cancer in Switzerland: a census-based cohort study. BMC Cancer. 2020;20:819.

6. Simon-Delso N, Amaral-Rogers V, Belzunces LP, Bonmatin JM, Chagnon M, Downs C, et al. Systemic insecticides (neonicotinoids and fipronil): trends, uses, mode of action and metabolites. Environ Sci Pollut Res. 2015;22:5-34

7. Humann-Guilleminot S, Binkowski ŁJ, Jenni L, Hilke G, Glauser G, Helfenstein $F$. A nation-wide survey of neonicotinoid insecticides in agricultural land with implications for Agri-environment schemes. J Appl Ecol. 2019;56:1502-14.

8. Humann-Guilleminot S, Clément S, Desprat J, Binkowski ŁJ, Glauser G, Helfenstein F. A large-scale survey of house sparrows feathers reveals ubiquitous presence of neonicotinoids in farmlands. Sci Total Environ. 2019;660:1091-7.

9. Chen M, Tao L, McLean J, Lu C. Quantitative analysis of Neonicotinoid insecticide residues in foods: implication for dietary exposures. J Agric Food Chem Am Chem Soc. 2014;62:6082-90.

10. Mitchell EAD, Mulhauser B, Mulot M, Mutabazi A, Glauser G, Aebi A. A worldwide survey of neonicotinoids in honey. Science. 2017;358:109-11.

11. Goulson D. REVIEW: an overview of the environmental risks posed by neonicotinoid insecticides. J Appl Ecol. 2013;50:977-87.

12. Dwyer JB, MCQuown SC, Leslie FM. The dynamic effects of nicotine on the developing brain. Pharmacol Ther. 2009;122:125-39.

13. Lloyd GK, Williams M. Neuronal nicotinic acetylcholine receptors as novel drug targets. J Pharmacol Exp Ther;United States. 2000;292:461-7.

14. Tomizawa M, Casida JE. Selective toxicity of neonicotinoids attributable to specificity of insect and mammalian nicotinic receptors. Annu Rev Entomol;United States. 2003;48:339-64.
15. Yang W, Carmichael SL, Roberts EM, Kegley SE, Padula AM, English PB, et al. Residential agricultural pesticide exposures and risk of neural tube defects and orofacial clefts among offspring in the San Joaquin Valley of California. Am J Epidemiol. 2014;179:740-8.

16. Marfo JT, Fujioka K, Ikenaka Y, Nakayama SMM, Mizukawa H, Aoyama $Y$, et al. Relationship between urinary N-Desmethyl-Acetamiprid and typical symptoms including neurological findings: A prevalence casecontrol study. PLoS One;Public Library of Science. 2015;10:1-19.

17. Keil AP, Daniels JL, Hertz-Picciotto I. Autism spectrum disorder, flea and tick medication, and adjustments for exposure misclassification: the CHARGE (CHildhood autism risks from genetics and environment) case-control study. Environ Health Glob Access Sci Source. 2014;13:3.

18. Carmichael SL, Yang W, Roberts E, Kegley SE, Brown TJ, English PB, et al. Residential agricultural pesticide exposures and risks of selected birth defects among offspring in the San Joaquin Valley of California. Birt Defects Res A Clin Mol Teratol;United States. 2016;106:27-35.

19. Ichikawa G, Kuribayashi R, Ikenaka Y, Ichise T, Nakayama SMM, Ishizuka $\mathrm{M}$, et al. LC-ESI/MS/MS analysis of neonicotinoids in urine of very low birth weight infants at birth. PLoS One. Public Library of Science. 2019;14:1-11.

20. Annabi E, Salem IB, Abid-Essefi S. Acetamiprid, a neonicotinoid insecticide, induced cytotoxicity and genotoxicity in PC12 cells. Toxicol Mech Methods. 2019;29:580-6.

21. Chakroun S, Ezzi L, Grissa I, Kerkeni E, Neffati F, Bhouri R, et al. Hematological, biochemical, and toxicopathic effects of subchronic acetamiprid toxicity in Wistar rats. Environ Sci Pollut Res. 2016;23:25191-9.

22. Erdemli ME, Zayman E, Erdemli Z, Gul M, Gul S, Gozukara BH. Protective effects of melatonin and vitamin $E$ in acetamiprid-induced nephrotoxicity. Environ Sci Pollut Res Int. 2020;27:9202-13.

23. Di Prisco G, lannaccone M, lanniello F, Ferrara R, Caprio E, Pennacchio $F$, et al. The neonicotinoid insecticide Clothianidin adversely affects immune signaling in a human cell line. Sci Rep. 2017;7:13446.

24. Cimino AM, Boyles AL, Thayer KA, Perry MJ. Effects of Neonicotinoid pesticide exposure on human health: A systematic review. Environ Health Perspect. 2017;125:155-62.

25. Zhang T, Song S, Bai X, He Y, Zhang B, Gui M, et al. A nationwide survey of urinary concentrations of neonicotinoid insecticides in China. Environ Int. 2019;132:105114.

26. Osaka A, Ueyama J, Kondo T, Nomura H, Sugiura Y, Saito I, et al. Exposure characterization of three major insecticide lines in urine of young children in Japan-neonicotinoids, organophosphates, and pyrethroids. Environ Res Netherlands. 2016;147:89-96.

27. Ospina M, Wong L-Y, Baker SE, Serafim AB, Morales-Agudelo P, Calafat AM. Exposure to neonicotinoid insecticides in the U.S. general population: data from the 2015-2016 national health and nutrition examination survey. Environ Res. 2019;176:108555.

28. Ikenaka Y, Miyabara Y, Ichise T, Nakayama S, Nimako C, Ishizuka M, et al. Exposures of children to neonicotinoids in pine wilt disease control areas. Environ Toxicol Chem. 2019;38:71-9.

29. Wang H, Yang D, Fang H, Han M, Tang C, Wu J, et al. Predictors, sources, and health risk of exposure to neonicotinoids in Chinese school children: A biomonitoring-based study. Environ Int. 2020;143:105918.

30. Bonmatin J-M, Mitchell EAD, Glauser G, Lumawig-Heitzman E, Claveria $F$, van Lexmond MB, et al. Residues of neonicotinoids in soil, water and people's hair: A case study from three agricultural regions of the Philippines. Sci Total Environ. 2021;757:143822.

31. Fuke C, Nagai T, Ninomiya K, Fukasawa M, Ihama Y, Miyazaki T. Detection of imidacloprid in biological fluids in a case of fatal insecticide intoxication. Leg Med Tokyo Jpn Ireland. 2014;16:40-3.

32. Warren KE. Beyond the Blood:Brain Barrier: The Importance of Central Nervous System (CNS) Pharmacokinetics for the Treatment of CNS Tumors, Including Diffuse Intrinsic Pontine Glioma. Front Oncol. 2018;8:239.

33. Kammoun S, Mulhauser B, Aebi A, Mitchell EAD, Glauser G. Ultra-trace level determination of neonicotinoids in honey as a tool for assessing environmental contamination. Environ Pollut. 2019;247:964-72.

34. Pearson MA, Lu C, Schmotzer BJ, Waller LA, Riederer AM. Evaluation of physiological measures for correcting variation in urinary output: implications for assessing environmental chemical exposure in children. J Expo Sci Environ Epidemiol. 2009;19:336-42. 
35. R Core Team. R: A language and environment for statistical computing [Internet]. R Lang. Environ. Stat. Comput. R Found. Stat. Comput. Vienna Austria. [cited 2021 Jul 23]. Available from: https://www.R-project.org/.

36. Chambers J, Chambers JM. Linear models. Chapter 4 of Statistical Models in S. In: Chambers JM, Hastie TJ, editors. Wadsworth \& Brooks/Cole, vol. 1992; 1992

37. Kassambara A. ggpubr:"ggplot2"Based Publication Ready Plots. [Internet]. R Package Version 040. 2020 [cited 2021 Jul 23]. Available from: https:// CRAN.R-project.org/package $=$ ggpubr.

38. Wang A, Mahai G, Wan Y, Yang Z, He Z, Xu S, et al. Assessment of imidacloprid related exposure using imidacloprid-olefin and desnitro-imidacloprid: Neonicotinoid insecticides in human urine in Wuhan, China. Environ Int. 2020;141:105785.

39. Li AJ, Martinez-Moral M-P, Kannan K. Variability in urinary neonicotinoid concentrations in single-spot and first-morning void and its association with oxidative stress markers. Environ Int. 2020;135:105415.

40. Xu M, Zhang Z, Li Z, Kan S, Liu Z, Wang D, et al. Profiles of neonicotinoid insecticides and characteristic metabolites in paired urine and blood samples: partitioning between urine and blood and implications for human exposure. Sci Total Environ. 2021;773:145582.

41. Thompson DA, Lehmler H-J, Kolpin DW, Hladik ML, Vargo JD, Schilling $\mathrm{KE}$, et al. A critical review on the potential impacts of neonicotinoid insecticide use: current knowledge of environmental fate, toxicity, and implications for human health. Env Sci Process Impacts. The Royal Society of Chemistry. 2020;22:1315-46.

42. Bennett B, Workman T, Smith MN, Griffith WC, Thompson B, Faustman EM. Characterizing the neurodevelopmental pesticide Exposome in a Children's agricultural cohort. Int J Environ Res Public Health. 2020;17 Available from: https://www.mdpi.com/1660-4601/17/5/1479.

43. Nau R, Sörgel F, Eiffert H. Penetration of drugs through the bloodcerebrospinal fluid/blood-brain barrier for treatment of central nervous system infections. Clin Microbiol Rev. 2010;23:858-83.

44. Ek CJ, Dziegielewska KM, Habgood MD, Saunders NR. Barriers in the developing brain and Neurotoxicology. Neurotoxicology Netherlands. 2012;33:586-604

45. Maitre L, de Bont J, Casas M, Robinson O, Aasvang GM, Agier L, et al. Human early life Exposome (HELIX) study: a European population-based exposome cohort. BMJ Open. 2018;8 Available from: https://bmjopen. bmj.com/content/8/9/e021311.

\section{Publisher's Note}

Springer Nature remains neutral with regard to jurisdictional claims in published maps and institutional affiliations.

Ready to submit your research? Choose BMC and benefit from:

- fast, convenient online submission

- thorough peer review by experienced researchers in your field

- rapid publication on acceptance

- support for research data, including large and complex data types

- gold Open Access which fosters wider collaboration and increased citations

- maximum visibility for your research: over $100 \mathrm{M}$ website views per year

At BMC, research is always in progress.

Learn more biomedcentral.com/submissions 